\title{
Students Selection for University Course Admission at the Joint Admissions Board (Kenya) Using Trained Neural Networks
}

\author{
Franklin Wabwoba \\ Masinde Muliro University of \\ Science and Technology, \\ Kakamega, Kenya
}

fwabwoba@gmail.com

\author{
Fullgence M. Mwakondo, \\ Mombasa Polytechnic \\ University College, \\ Mombasa, Kenya
}

mwakondopoly@gmail.com

\section{Executive Summary}

Every year, the Joint Admission Board (JAB) is tasked to determine those students who are expected to join various Kenyan public universities under the government sponsorship scheme. This exercise is usually extensive because of the large number of qualified students compared to the very limited number of slots at various institutions and the shortage of funding from the government. Further, this is made complex by the fact that the selections are done against a predefined cluster subjects vis a vis the student's preferred and applied for academic courses. Minimum requirements exist for each course and only students having the prescribed grades in specific subjects are eligible to join that course. Due to this, students are often admitted to courses they consider irrelevant to their career prospects and not their preferred choices.

This process is tiresome, costly, and prone to bias, errors, or favour, leading to disadvantaging innocent students. This paper examines the potential use of artificial neural networks at the JAB for the process of selecting students for university courses. Based on the fact that Artificial Neural Networks (ANNs) have been tested and used in classification, the paper explains how a trained neural network can be used to perform the students' placement effectively and efficiently. JAB will be able, therefore, to undertake the students' placement thoroughly and be able to accomplish it with minimal wastage of time and resources respectively without having to utilise unnecessary effort. The paper outlines how the various metrics can be coded and used as input to the ANNs. Ultimately, the paper underscores the various merits that would accompany the adoption of this technique. By making use of neural networks in the university career choices, student placement at JAB will enhance the chances of students being placed into courses they prefer as part of their career choice. This is likely to motivate the students, making them work harder and leading to improved performance and improved completion rate. The ANN application may also reduce the

Material published as part of this publication, either on-line or in print, is copyrighted by the Informing Science Institute. Permission to make digital or paper copy of part or all of these works for personal or classroom use is granted without fee provided that the copies are not made or distributed for profit or commercial advantage AND that copies 1) bear this notice in full and 2) give the full citation on the first page. It is permissible to abstract these works so long as credit is given. To copy in all other cases or to republish or to post on a server or to redistribute to lists requires specific permission and payment of a fee. Contact HPublisher@InformingScience.orgH to request redistribution permission. cost spend on the application processing and the time the applicants have to wait for the outcome. The ANN application could further increase the chances of high quality applicants getting admission to career courses for which they qualify. 
Keywords: neural networks, university admission, cluster subjects, minimum requirements, university courses, selection.

\section{Introduction}

Student selection for university courses in Kenya is an activity that is performed by the Joint Admissions Board (JAB) each year. In this process students are allocated courses of their choice according to their performance in specific subjects. Minimum requirements exist for each course and only students having the prescribed grades in specific subjects are eligible to join a particular course. This activity may be costly and prone to bias, errors, or favor, leading to disadvantaging some students.

Admission requirements for these universities are dynamic and keep fluctuating each year depending on the overall performance of students. Course requirements have to be revised every year in order to scale the number of student admissions according to limited university slots. This activity can be tedious, time consuming and calls for systems that are also dynamically changing to manage the task. The approach is also subject to abuse by insiders within JAB and inappropriate use of resources. Information and Communication Technology (ICT) has the potential to provide tools / technologies for building suitable systems for preventing insider abuses and ensuring appropriate use of the limited resources available (Sodiya \& Onashoga, 2009). In as much as ICT is changing in itself, it also makes enhancements in technology that can cope with the changes from other applications. This would allow ICT applications to cope with the changing needs like those arising out of the dynamically changing course requirements for university admissions. Innovations in the field of Information Technology (IT) continue to increase at an ever spiraling rate; advances in operating systems, software, communication devices, and methodologies are renovating the inventory of IT products on a near daily basis (Gillard, Bailey, \& Nolan, 2008).

As a result of the current system students have been assigned to courses they did not apply for. They later find the courses that were assigned to them beyond their scope, get overwhelmed, and keep on re-sitting for exams ("Joint Board Should Review," 2009). This may result in many opting to forgo those courses in favour of courses they preferred in the private universities both in Kenya and abroad or self sponsored programmes in Kenyan public universities for those who have finances. The poor, who cannot afford financing self-sponsored university education, will therefore just lose out. It is estimated that only $20 \%$ of the qualified students eventually get admitted into local universities every year (Munavu, Ogutu, \& Wasanga, 2008). The selection process for university admission has denied many capable students a chance in a public university.

This paper discusses the applicability of artificial neural networks in the process of selecting students for admission to any of the public universities. The paper intends to reveal that artificial neural networks can be used to identify the right university course for students respectively within their chosen options. It is hoped that it motivates the concerned parties to look at possibilities of making use of and working out modalities of employing the use of artificial intelligence in the selection process.

\section{Joint Admission Board (JAB)}

Public universities (in Kenya) and their constituent colleges conduct a joint admission exercise to their universities under a common framework called the Joint Admission Board (JAB). This is mainly to ensure that access to university education is based on academic merit for institutionalbased undergraduate students (This excludes students who are admitted to distance education / self-sponsored programmes). Students admitted through the board get funding from the government. This admission exercise is performed annually before the start of each academic year for 
candidates who have sat the Kenya Certificate of Secondary Education (KCSE) examination the previous year.

During the admission process, priority is given to an applicant's first choice. The applicant's second to fourth choices are considered where vacancies still exist (Joint Admissions Board, 2009). JAB makes the choice for a student in cases where vacancies which exist do not match with the student's choice. However, due to limited capacity in popular programs, some qualifying students with the required cut-off points end up in courses that might not have been their preferred choices (Munavu et al., 2008).

\section{Challenges JAB is Facing}

JAB faces a number of challenges in carrying out students' placement into courses and universities. The major challenges include poor student turnout after selection, the mode of selection for admission that is deterministic, increased enrolment due to double intake, and addressing gender inequality.

\section{Poor student turnout after selection}

Some applicants selected by JAB are unable to report for their selected programmes due to financial problems. This may result to poor turnout of students which amounts to classes with no quorum. Students from poor backgrounds face a hard task getting loans, while the rich find it easy (“Joint Board Should Review,” 2009).

\section{Mode of selection for admission that is deterministic}

JAB's mode of admission relying only on exam results may deny opportunities to capable applicants who did not perform well in the exam, not because they are not bright, but due to serious circumstances during exam time or disadvantages due to regional and school imbalances. Access to higher education in Kenya is still largely dependent on performance in KCSE examinations, since this is used as the standardized selection criteria. During the admission process applicants who are not able to secure a place into the programmes of their preferred choice are offered courses they might consider irrelevant to their career aspirations (Munavu et al., 2008).

\section{Increased enrolment due to double intake}

Extensive expansion of higher education in Kenya in terms of the number of students demanding access against limited university spaces has led to stiff competition and constant rise of entry requirements.

\section{Gender inequality}

Given the fact that there should be equal representation of both sexes at all levels, female applicants have to be given special and fair selection in relation to their male counterparts. This should be done especially in the sciences, mathematics, and technology oriented subjects where gender disparities have often been observed (Chacha, 2004). Studies indicate that there is a higher number of female students than male students in private universities. This could be due to the existing gender imbalance in public universities in favor of male students, leading to more female students seeking university education at private universities. Female students in public universities comprise about $40 \%$ of the total number of students (Munavu et al., 2008). 


\section{Artificial Neural Networks (ANN)}

\section{Background}

Neural networks are composed of simple elements operating in parallel. These elements are inspired by biological nervous systems. As in nature, the connections between elements largely determine the network function (Demuth, Hagan, \& Beale, 2009). An artificial neural network (ANN) consists of a collection of processing elements that are highly interconnected and transform a set of inputs into a set of desired outputs. The result of the transformation is determined by the characteristics of the elements and the weights associated with the interconnections among them. By modifying the connections between the nodes the network is able to adapt to the desired outputs (Cannady, 1998).

An ANN can be trained to perform a particular function by adjusting the values of the connections (weights) between elements. A pair of actual input and desired output is required to train the artificial neural network. Neural networks have been trained to perform complex functions in various fields, including pattern recognition, identification, classification, speech, vision, and control systems (Demuth et al., 2009). The neural network gains the experience initially by training the system to correctly identify pre-selected examples of the problem. The response of the neural network is reviewed and the configuration of the system is refined until the neural network's analysis of the training data reaches a satisfactory level. Training of the network is conducted until outputs of the network satisfy the desired target or until the network reaches desired performance as indicated by some error level (difference between output and desired target of network). Usually this error is formulated as mean square error (MSE) (Haryanto, Setiawan, \& Budiyono, 2007). In addition to the initial training period, the neural network also gains experience over time as it conducts analyses on data related to the students' course selection as per their preferred choice.

\section{Neurons}

A neuron is the building block of a neural network that consists of input links (from other neurons), an input function, an activation function, output, and output links (to other neurons). Input links provide activation to the neuron from other neurons. Each input link is assigned a weight which determines both the activation strength of connection and the sign. The stronger the activation strength the more likely it will be for the option to be connected to for further processing. A weighted sum is calculated for each neuron, $i$, and then the weighted sum of its entire input links, which are then used in the activation function in order to derive its output, denoted by $\mathrm{a}_{\mathrm{i}}$ (Bigelow, 2009). The activation function is designed such that it meets two conditions. Firstly, the neuron should be active (with output near +1 ) when the correct inputs are given to it, and inactive (with output close to 0) when the incorrect inputs are given. Secondly, the activation function needs to be nonlinear, to keep the entire neural network from becoming a simple linear function. There are two possible choices of activation functions: threshold and sigmoid functions (Lin, Zhang, \& Thibault, 2009). The sigmoid function is differentiable and therefore useful for a weight-learning algorithm. Figure 1 shows a neuron, that has $\mathrm{N}$ inputs $\left(\mathrm{u}_{1}, \mathrm{u}_{2}, \ldots, \mathrm{u}_{\mathrm{j}} \ldots, \mathrm{u}_{\mathrm{n}}\right)$. The inputs are each assigned a weight $\mathrm{W}\left(\mathrm{w}_{1}, \mathrm{w}_{2}, \ldots, \mathrm{w}_{\mathrm{j}}, \ldots, \mathrm{w}_{\mathrm{n}}\right)$ having a threshold denoted by,$\theta$, which allows for optimization of the initial start values thus giving an activation function of

$$
a_{i}=\sum_{j=1}^{n}\left(u_{i} w_{j}\right)+\theta
$$




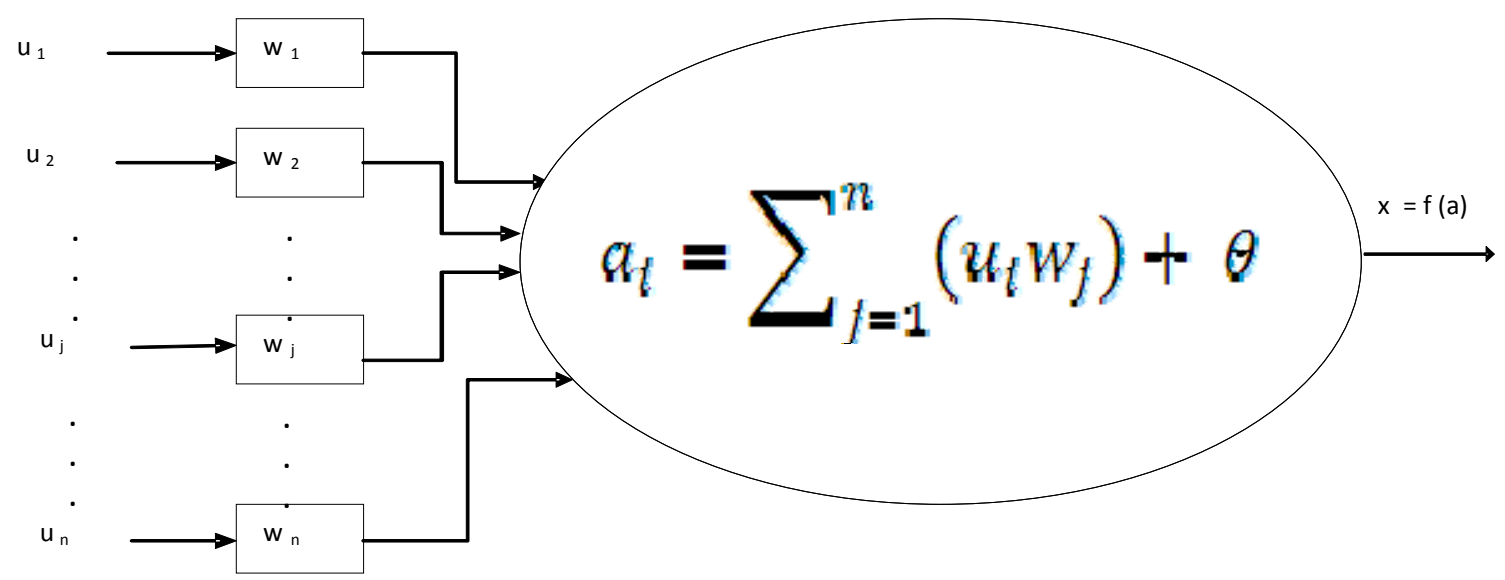

Figure 1: Neuron showing input, weight, input function, activation function, and output.

Notice that in addition to the inputs and weights from the other neurons in the network, each neuron also gets a bias. This sets the actual threshold, $\theta$, for the neuron. This allows us to adjust the threshold of "a" away from 0 , where it would normally be (Bigelow, 2009). The output value of the neuron is a function of its activation $x=f(a)$.

\section{Process of Artificial Neural Networks}

ANNs are mathematical entities or models based on the basic building block known as the artificial neuron and usually modeled after the biological neuron found in the brain. The artificial neurons making up the ANN are distributed processors and operate in parallel. In fact, an ANN resembles the brain in two aspects: it is able to acquire knowledge through learning and to store the knowledge through interconnection strengths known as synaptic weights (Haykin, 2004).

The ANN process basically consists of input data intended to be processed in a certain desired way, computational processors comprising several neurons that are capable of extracting important features contained in the input data, output of computational processes, expected target values, and control mechanism for adjusting the parameters values (weights and biases) not determined by a students performance in the exam. Figure 2 below shows the conceptual model of ANN highlighting its main elements.

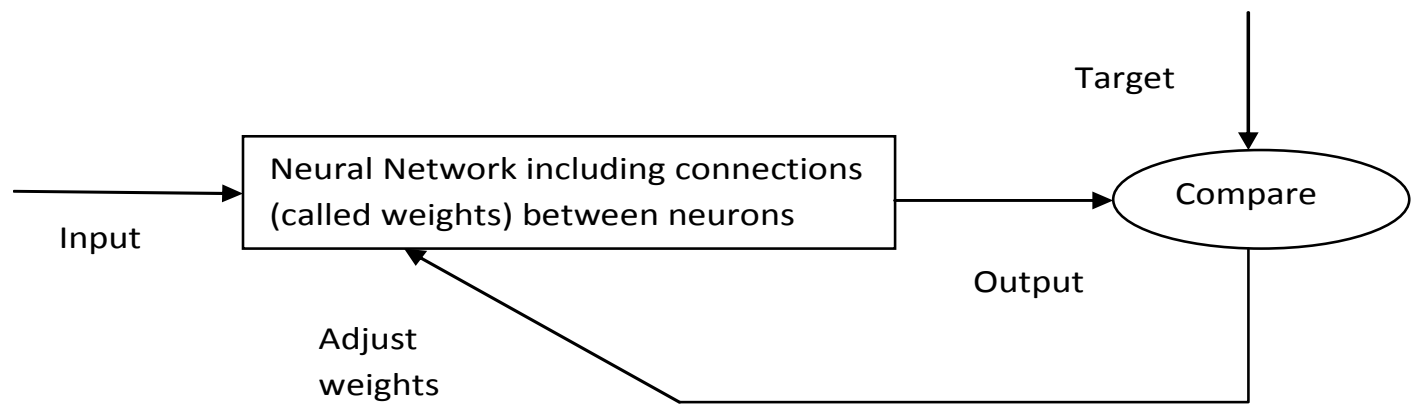

Figure 2: Conceptual model of an artificial neural network

The artificial neurons in ANNs are arranged in layers, each layer providing different functions to the ANN, e.g., an input layer consisting of input neurons, an output layer consisting of output neurons, etc. 


\section{Types of artificial neural networks}

ANNs may be single layered or multiple layered.

\section{Single-layered and multiple-layered ANNs}

A neural network with all of its inputs connected directly to the outputs is called a single-layer network, or a perceptron network. While a single artificial neuron is not able to do some tasks, the problem is overcome by connecting the outputs of some neurons as input to the others, so as to constitute a neural network. Suppose we have connected many artificial neurons we assign indices to the different neurons in the system to discriminate between them. In the case of the $i^{\text {th }}$ activation neuron, for example, the formulas are modified as follows:

$\mathrm{a}_{\mathrm{i}}=\theta_{\mathrm{i}}$ where $\theta_{\mathrm{i}}=\mathrm{b}_{\mathrm{i}}$ in Figure 3 below.

Equation 2

Figure 3 below is a diagram showing a single-layer neural network with $\mathbf{s}$ neurons.

$\begin{array}{lll}\text { Input vector } & = & \left\{\mathrm{P}_{1}, \mathrm{P}_{2}, \mathrm{P}_{3}, \ldots, \mathrm{P}_{\mathrm{R}}\right\} \\ \text { Input link [weights] } & = & \left\{\mathrm{w}_{\mathrm{ji}}\right\} \\ \text { Input function } & = & \sum \\ \text { Activation function } & = & f \\ \text { Output } & = & \left\{\mathrm{a}_{1}, \mathrm{a}_{2}, \mathrm{a}_{3}, \ldots, \mathrm{a}_{\mathrm{s}}\right\} \\ \text { Bias } & = & \left\{\mathrm{b}_{1}, \mathrm{~b}_{2}, \mathrm{~b}_{3}, \ldots, \mathrm{b}_{\mathrm{s}}\right\}\end{array}$

Where $\mathrm{R}$ is the number of elements in the vector and $\mathrm{S}$ is the number of neurons in layer

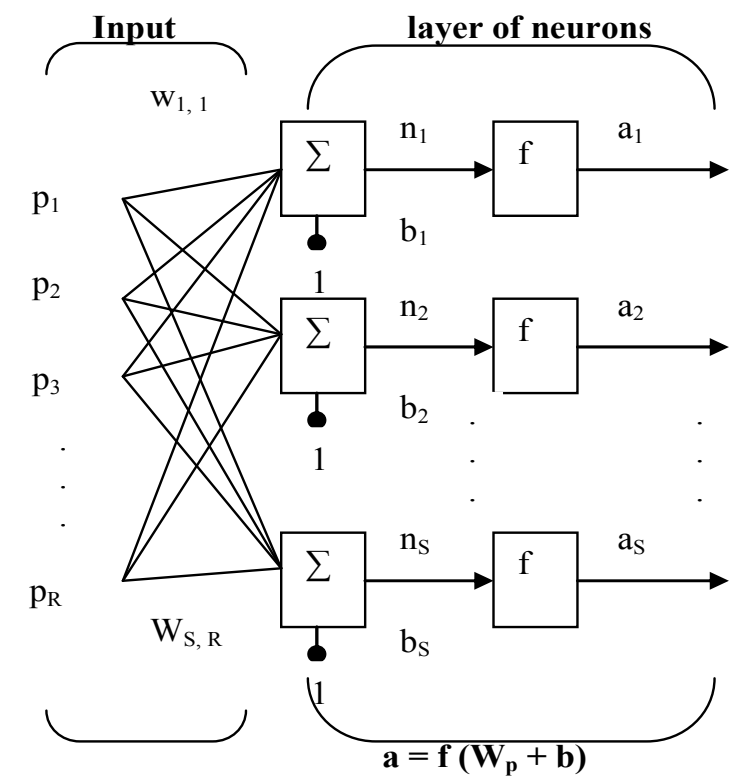

Figure 3: Single-layered network

In the equation above, $\mathrm{W}_{\mathrm{p}}$ is the weight cluster point. How this is normally obtained will be explained in equation 3 in a later section. The variable $\mathbf{b}$ is an assigned factor that varies with each year.

A neural network with layers in between the input layer and the output layer is known as multiple-layer network or multiple-layer perceptron (MLP). The layers in between are known as hidden layers. A multiple-layer perceptron can have one or more hidden layers and these layers are not directly accessible to the environment. Figure 4 represents a multiple-layer network with three layers each with three neurons. Layer 1 represents the input layer, layer 3 is output layer and layer 
2 represents the hidden layers for the purpose of illustration. There could be many hidden layers in a system.

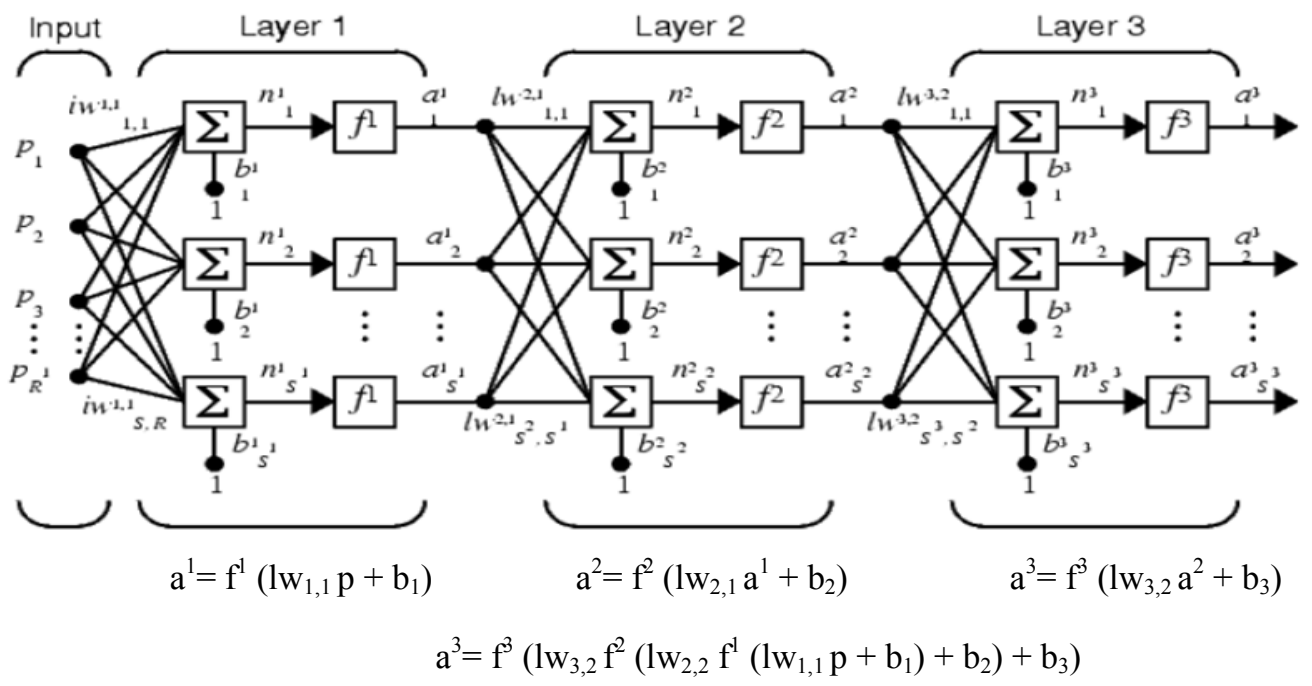

Figure 4: Multiple-layer network with three layers

$\mathrm{W}_{\mathrm{ij}}$ is the weight point for the career choice with i representing the level of the layer and $\mathrm{j}$ the level of the layer giving in in-feed. The activation function above is an expansion of the function given in Figure 3.

\section{Feedforward and re-current network}

A feed-forward network represents a function of its current input, needing no internal state other than the weights themselves. A recurrent network, however, has to feed its outputs back into its own inputs. This means that the activation levels of the network will form a dynamic system that can exhibit numerous behaviors, including reaching a stable equilibrium or exhibiting oscillations or even chaotic behavior (Bigelow, 2009).

\section{Learning and training artificial neural networks}

An ANN has to be trained in order to learn and gain knowledge required to do a certain task. Training of ANNs involves devising some learning rules or procedures for modifying weights and biases of a network. These learning rules will be the training algorithm for the neural network. Learning can be classified into the following forms. (1) Supervised learning in which the learning rule is provided with examples of a set of corresponding input-output (desired target) pair. As the inputs are applied to the system the outputs produced are compared with the target and the learning rule is used to adjust the weights and the biases so as to move the network output closer to the target. (2) Unsupervised learning happens when no targets are used to modify network weights and biases. In this case network weights and biases are modified using inputs only.

\section{The Application of Artificial Neural Networks in Classification}

Classification is one of the most frequently encountered decision making tasks of human activity. A classification problem occurs when an object needs to be assigned into a predefined group or class based on a number of observed attributes related to that object (Zhang, 2000). Neural networks have been used in many application areas, such as business, science, and industry. Major types of applications include bankruptcy prediction, handwriting recognition, speech recognition, medical diagnosis, product inspection, and bond rating. This only serves to show that applying neural networks to the selection of students for university admission could be possible. 
Classification is the process of associating a given input vector with a target vector. An artificial neural network uses two approaches of classification. One approach is used when there are only two classes to classify the input vector. The other approach is used when there are several classes to classify the input vector. An artificial neural network with only two output neurons is used in the former approach while the later approach uses more than two output neurons.

\section{Artificial Neural Networks (ANN) in Student Selection for University Admission}

Substantial research has been done in the use of neural networks for pattern recognition or classification. Many studies have been conducted on the application of artificial neural networks in computer security, detection of computer intrusion and detection of computer viruses, image detection, and fingerprint recognition. However, hardly any research exists in the area of student selection for university admission.

Research activities in neural classification have established that neural networks are a promising alternative to various conventional classification methods (Zhang, 2000). Traditional statistical classification methods are becoming unpopular because of their high reliance on the assumptions made. On the other hand, current rule-based systems, expert systems, are weak in their ability to deal with the uncertainties inherent in real-world information and lack the ability to generalize to novel problems (Goodman, Higgins \& Miller, 1992). One important advantage is that neural networks are nonlinear models, which makes them flexible for modeling complex relationships in the real world (Zhang, 2000).

While there is an increasing need for a more accurate system capable of identifying the academic qualities/characteristics for students to join suitable university courses, there is currently no applied alternative to rule-based systems. Rule-based systems can be relatively effective if the exact characteristics (or academic qualities) of students are known. However, these academic characteristics/qualities of students are ever changing depending on the qualities of examinations, marking, and teaching and other socio economic factors. Rule-based systems cannot accurately identify a variety of students who qualify to be selected for certain university courses as a large group of students attain a minimum grade to join universities. The qualities of examinations and teaching standards are also changing constantly. As a result, many students are misplaced or rejected.

The quality of examinations, teaching standards, and curriculum may affect the academic qualities/characteristics of a student required for a particular course. Therefore, the process of selecting students requires a flexible system that is capable of analyzing vast volumes of student requirements in a less structured manner than the rule-based system. Artificial neural network system could potentially address many of the problems experienced with rule-based systems more effectively.

\section{Proposed Neural Network Architecture for Selecting Students}

Based on the above discussion, it is therefore likely that the application of ANNs may provide more efficient and effective students placement into courses and universities. The architecture involves the problem formulation, an appropriate strategy design, an implementation plan to be worked out, and an assessment of the merit and demerits of using ANNs, as will be discussed below. 


\section{Problem Formulation}

The problem formulation may begin by modeling the information into a structure that would form the data set which is used as input to the network training. The model has to be capable of representing most of the possible causes / factors / characteristics of a student's performance in the final examination. The final result / grade / score of a student is modeled as the performance of the student consisting of the overall grade or mark and the subject areas in which the student performed well. In order to obtain the data set as input for the network training, values of student performance details are obtained. The values represent subjects failed or passed between 1 and 12 inclusive, where 1 represents the extreme fail and 12 represents the best pass. These values are derived from seven subjects: English, Kiswahili, Mathematics, two sciences subjects, one humanity subject, and the subject left over or the best subject chosen from those left over if the student took more than seven subjects.

\section{Design Strategy}

The factors listed below have been proposed for now to determine (are related to or connected to) the student performance for selection to a university course. The data for these factors can easily be collected.

1. overall grade (student's mean grade)

2. cluster aggregate (student aggregate score for the four subjects that are pre-requisite for being admitted to a particular university career course)

3. subjects done (subjects actually examined)

Any combination of the above factors may be considered to contribute to a student's performance and qualification to a particular university course. The number of these factors and the actual cause of student's performance (failure or success) will be assumed as random and may be due to a multiple of these factors. The data for the above factors will be collected from Kenya National Examination Council (KNEC) or JAB for the neural network training. The target for our neural network system will be either a pass or a fail which will be denoted by 1 or 0 respectively.

The data set collected from KNEC or JAB has to be pre-processed so that the overall grade and cluster aggregate are in point form. All the subjects examinable are listed for input and a 1 or 0 will be used to represent subject done or not done by the student respectively. The target will consist of unique binary codes for each university course that a student can qualify for, e.g., 1000000000 for Bsc. Electrical Engineering, 0100000000 for Bsc. Civil Engineering, etc. Requirements for each course will be unique. Table 1 summarizes the input and target data layout. Table 2 summarizes the target codes for the university courses.

Table 1: Sample table summarizing input and target data layout

\begin{tabular}{|c|c|c|c|c|c|}
\hline Performance factors & Students' pe & formance det & & & \\
\hline & 1 & 2 & 3 & 4 & \\
\hline 1. Overall grade & 11 & 10 & 9 & 8 & $\ldots$ \\
\hline 2. Cluster aggregate & 9 & 8 & 7 & 8 & $\ldots$ \\
\hline 3. Mathematics & 1 & 1 & 1 & 1 & $\ldots$ \\
\hline 4. English & 1 & 1 & 0 & 1 & $\ldots$ \\
\hline 5. Physics & 1 & 1 & 1 & 1 & $\ldots$ \\
\hline f.................... & ...... & ...... & ...... & ...... & $\ldots \ldots$ \\
\hline TARGET & 1000000000 & 0100000000 & 0010000000 & 0001000000 & $\ldots$ \\
\hline
\end{tabular}

The column headers indicate the unique student codes, here shown as 1, 2, 3, 4, ... Below each student code are the performance details against the row headers representing performance fac- 
tors, here indicated as overall grade, cluster aggregate, Mathematics, English, Physics, etc.. The overall grade for a student is the mean score/points of all the subjects done and can be any of the following alphabetical grades (A, A-, B+, B, B-, C+, C, C-, D+, D, D-, E) whose equivalent numerical points (scores) as shown in the table are $(12,11,10,9,8,7,6,5,4,3,2,1)$ respectively. The cluster aggregate of a student is a total score/points in those subjects indicated as core for a particular course. There are normally four cluster subjects with each having a possible maximum of 12 , hence giving a maximum sum points of 48 . The total score is obtained by summing the actual points obtained in each of the cluster subjects. The cluster aggregate can therefore range from 0 to any number depending on the number of core subjects. The possible subjects to be done are indicated as Mathematics, English, Physics, etc. and are awarded a 1 if done and passed as per requirement (meets minimum course requirement) by the student and a 0 if not. According to the Joint Admissions Board (2009), the value of $\mathrm{W}_{\mathrm{p}}$ is evaluated by the formula below:

$w_{p}=\sqrt{\left(\frac{c}{48} \times \frac{d}{84}\right)} \times 48$

where $\mathbf{C}$ is the total points from the four cluster subjects for the chosen career and $\mathbf{d}$ is the total points gained out of the overall performance of the students with 48 being the maximum possible points one can get from cluster subjects while 84 is the maximum points one can get from the seven subjects ranked in the exam.

Table 2: Sample course code (target) data

\begin{tabular}{|r|l|}
\hline Course name & Course code (target) \\
\hline $1 . \quad$ Bsc. Electrical and Electronics Engineering & 1000000000 \\
\hline $2 . \quad$ Bsc. Agricultural Engineering & 0100000000 \\
\hline $3 . \quad$ Bsc. Civil Engineering & 0010000000 \\
\hline $4 . \quad$ Bsc. Science & 0001000000 \\
\hline 5. Bsc. Mechanical Engineering & 0000100000 \\
\hline n. B........ & $\ldots \ldots \ldots \ldots \ldots$ \\
\hline
\end{tabular}

\section{Significance of the Design Strategy}

The design strategy is simple to develop and easy to understand. The codes are simple and easy to construct.

The design strategy considers both the preference of the applicant and his/her academic potential before providing a suitable alternative based only on academic potential.

The design strategy provides room for the applicants to give as many course preferences as they wish, since this implies only adding an additional column in Table 3.

\section{Implementation Plan}

The above design strategy can be implemented using a supervised multiple layer perceptron (MLP) neural network architecture because of its simplicity. The neural network will have as many output nodes as the size of the target code, one for each bit. The number of input nodes will 
be equivalent to the number of performance factors. The sigmoid function will be used as the activation for the neural network. The MATLAB platform will be used for implementing, training and testing the neural network. The number of hidden layers, and the number of nodes in the hidden layers, will be determined using trial and error process. Special weights can be allocated to the following categories of students:

$\begin{array}{lll}\text { Male applicant } & = & \mathrm{w}_{1} \\ \text { Female applicant } & = & \mathrm{w}_{2} \\ \text { Disabled applicant } & = & \mathrm{w}_{3}\end{array}$

The implementation plan can be achieved using the model below.

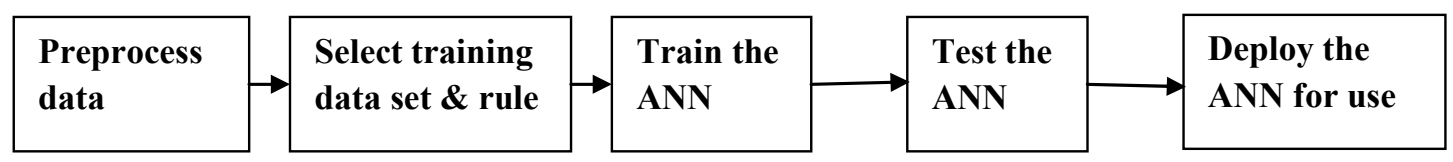

Figure 5: Implementation model

Preprocessing is required to calculate the cluster aggregate for each course that a student has selected as his/her preferred options, i.e., $1^{\text {st }}$ choice, $2^{\text {nd }}$ choice, $3^{\text {rd }}$ choice, etc.; assuming each student made three course choices for possible university admission, the preprocessed data will be presented in the form shown in Table 3 below. The row labeled as 'COURSE CHOSEN' will be filled with student selected courses for $1^{\text {st }}$ choice, $2^{\text {nd }}$ choice and $3^{\text {rd }}$ choice in that order of preference. Row labeled as 'ANN GENERATED' will be filled with values generated by the neural network.

Table 3: Sample table layout to be filled with students' data before processing

\begin{tabular}{|c|c|c|c|c|c|c|c|c|c|c|c|c|c|c|c|}
\hline \multirow{3}{*}{$\begin{array}{r}\text { Student's } \\
\text { code } \\
\text { Performance }\end{array}$} & \multicolumn{15}{|c|}{ STUDENTS' PERFORMANCE DETAILS } \\
\hline & \multicolumn{3}{|l|}{1} & \multicolumn{3}{|l|}{2} & \multicolumn{3}{|l|}{3} & \multicolumn{3}{|l|}{4} & \multicolumn{3}{|l|}{$\ldots$} \\
\hline & $1^{\text {st }}$ & $2^{\text {nd }}$ & $3^{\text {rd }}$ & $1^{\mathrm{st}}$ & $\begin{array}{l}2^{n} \\
d^{n}\end{array}$ & $3^{\text {rd }}$ & $1^{\mathrm{st}}$ & $\begin{array}{l}2^{n} \\
d^{n}\end{array}$ & $3^{\text {rd }}$ & $1^{\text {st }}$ & $\begin{array}{l}2^{n} \\
d^{n}\end{array}$ & $3^{\text {rd }}$ & $1^{\mathrm{st}}$ & $\begin{array}{l}2^{n} \\
d\end{array}$ & $3^{\text {rd }}$ \\
\hline 1. Overall grade & & & & & & & & & & & & & & & \\
\hline $\begin{array}{ll}\text { 2. } & \begin{array}{l}\text { Cluster } \\
\text { aggregate }\end{array}\end{array}$ & & & & & & & & & & & & & & & \\
\hline 3. Mathematics & & & & & & & & & & & & & & & \\
\hline 4. English & & & & & & & & & & & & & & & \\
\hline 5. Physics & & & & & & & & & & & & & & & \\
\hline ….............. & ‥ & ‥ & .. & $\cdots$ & ․ & .. & $\cdots$ & $\cdots$ & ‥ & ‥ & ‥ & $\cdots$ & ‥ & $\cdots$ & $\cdots$ \\
\hline $\begin{array}{l}\text { COURSE } \\
\text { CHOSEN }\end{array}$ & & & & & & & & & & & & & & & \\
\hline $\begin{array}{l}\text { ANN } \\
\text { GENERATED }\end{array}$ & & & & & & & & & & & & & & & \\
\hline
\end{tabular}


Before the ANN can be useful for any function it must be trained using a well selected set of training data and learning rules or algorithms. Table 1 (sample table summarizing input and target data layout) shall be used to collect a training data set relating to selection in the previous years. Given the multiple-layer perceptron (MLP) with a total number of synaptic weights, W, a rule of the thumb for selecting a training data set with sample size, $\mathrm{N}$, is

$$
N=O\left(\frac{W}{\varepsilon}\right)
$$

Equation 4

Where $O$ denotes "the order of," and $\mathcal{E}$ denotes the fraction of classification errors permitted on test data. For example, with an error of $10 \%$ the number of training examples needed should be about 10 times the number of synaptic weights in the network (Haykin, 2004). The training algorithm used with MLP is a back-propagation algorithm (Rumelhart, Hinton \& Williams, 1986; Werbos 1974) as cited by Haykin (2004). A back-propagation algorithm consists of two phases: a forward phase where the free parameters (weights and biases) are fixed as input is propagated forward, layer after layer, to finally produce the deviation or error and a backwards phase where the error or deviation signal is propagated through the network in the backwards direction during which the free parameters are adjusted to minimize the error.

Training the ANN involves using the MATLAB platform to run the training data set. The training data set is split into two subsets: the estimation data subset and the validation data subset. The estimation data subset is used for training the ANN model and the validation data subset is used for evaluating the model performance. The entire set is then used to fine tune the model. The training is repeated with a larger training data set and adjusting the number of neurons until a satisfying performance level of the model is reached. Finally, the model is tested with data that is different from the training data set.

When deployed for use, the input to ANN in the MATLAB platform will be a vector consisting of input performance details from each column in Table 3: (sample table layout to fill preprocessed student data before processing). The result of the processing for each column shall be recorded at the bottom of each column against the row indicated as ANN GENERATED. The student preferred course choices $\left(1^{\text {st }}\right.$ choice, $2^{\text {nd }}$ choice, $3^{\text {rd }}$ choice $)$ shall also be indicated against the COURSE CHOSEN row. At the end of the processing the two rows at the bottom of Table 3 , ANN GENERATED and COURSE CHOSEN will be compared for any matches. A student will be awarded an ANN generated course that matches his or her choice, starting with a choice with the highest preference downward. A student who does not get any matches to his or her choices will be advised to consider the other possible choices generated by the ANN.

\section{Advantages of Neural Networks in Student Selection}

A neural network use will speed up processing and it will be possible to process large volumes of student data within a very short time unlike the manual approach that takes a very long time, making students wait for one and a half years before they can join a university. As a result of this, JAB will save money and time in terms resources that would otherwise be needed to process large volumes of data. Universities will have the benefit of admitting motivated students as they will have had a better chance of selecting applicants into courses of their own choice. The students will have a shorter time to wait for their notification of admission to a university. It is more likely that students pursuing courses of their own choice will be better motivated to succeed.

A neural network is flexible and will be able to handle most cases where data might be missing due to data corruption, loss, or damage. This may mean that applicants will not get disqualified due to data corruption or loss and, instead, will remain assured of university admission. This may also mean that JAB will have fewer issues trying to recover lost or damaged data, while the uni- 
versities and the nation at large will not be affected so much when trying to adjust entry requirements and will not need to use a lot of manpower.

Neural networks provide a predictive capability of detecting cases where cheating occurred during the processing of student data. This ensures that applicants of low quality do not get admission to programmes for which they do not qualify and eventually risk failing. Both universities and students could be spared unnecessary embarrassment and disappointment, while the Government saves resources and eliminates losses by investing wisely to needy and deserving cases.

\section{Limiting Neural Networks in Student Selection}

Neural networks require accurate training using accurate training datasets and accurate training methods. This may be overcome by involving well trained data clerks and by applying verification methods to identify and remove any errors of transcription.

Neural networks require large volumes of data for training to ensure that the results are statistically significant. This may be overcome by using historical data accumulated at JAB over the past years.

The internal process of the artificial neural network is, however, more complex and less easy to reproduce and explain. It functions as a "black box" to a much larger extent than for traditional statistical methods (Rosini, 2000).

\section{Conclusion}

Substantial research has been done on the use of neural networks for pattern recognition or classification. Many research studies have been conducted on the application of artificial neural networks in computer security, detection of computer intrusion and detection of computer viruses, image detection, and fingerprint recognition. There is very little if any research that has been done in the area of student selection for university admission. There are many challenges faced by $\mathrm{JAB}$ in selecting students for university admission. These challenges arose due to the net effect of several random factors that play a role in shaping student performance. Student characteristics for university admission have been fluctuating making it very difficult to recognize required patterns. Neural networks could play a beneficial role in trying to resolve these challenges, especially given the several advantages they have. One such advantage is that universities are likely to have highly motivated students as these students will now be doing courses of their own choice. This may lead to improved chances of having a better graduate at the end of the training. This in itself will translate to improved quality of the manpower produced. It is also likely to reduce the costs and time spent on the processing the students placement. The time students normally spend out of learning could also be greatly reduced. The ANN application may reduce the cost spent on the application processing and the time the applicants have to wait for the outcome. The ANN application increases the chances for the applicants of high quality to get admission to career courses for which they are qualified.

Future work in this area should focus in incorporating more performance factors to be used as input data. These factors should include probabilistic elements that may be causing variation in student performance in examinations. Furthermore, other factors like poverty level, geographical location, the standard of the schooling, and family background are not the same for all students as some students are more disadvantaged than others. It might thus be valuable to incorporate these factors because they do affect overall performance of students. 


\section{References}

Bigelow, K. (2009). Lecture notes for AI (CS-482) Fall 2009, Lecture 24.

Cannady, J. (1998). Artificial neural networks for misuse detection. Retrieved February 17, 2010 from http://csrc.nist.gov/nissc/1998/proceedings/paperF13.pdf

Chacha, C. (2004). Reforming higher education in Kenya: Challenges, lessons and opportunities. The Interuniversity Council of East Africa at the State University of New York Workshop with Parliamentary Committee on Education, Science and Technology, Aug. 2004, Naivasha, Kenya. Retrieved on March 24 from http://www.iucea.org

Demuth, H., Hagan, M., \& Beale, M. (2009). MATLAB Neural Network Toolbox v. 4.0.4 User's Guide. Retrieved February 20, 2010 from http://www.mathworks.com/access/helpdesk/help/toolbox/nnet/ and http://www.mathworks.com/access/helpdesk/help/pdf_doc/nnet/nnet.pdf

Gillard, S., Bailey, D., \& Nolan, E. (2008). Ten reasons for IT educators to be early adopters of IT innovations. Journal of Information Technology Education, 7, 21-33. Retrieved from http://www.jite.org/documents/Vol7/JITEv7p021-033Gillard257.pdf

Goodman, R., Higgins, C., \& Miller, J. (1992). Rule-based neural networks for classification and probability estimation. Retrieved February 25, 2010 from http://id3490.securedata.net/rod/pdf/RG.Paper.JA27.pdf

Haryanto, I., Setiawan, J. \& Budiyono, A. (2007). Structural damage detection using randomized trained neural networks. ICIUS Bali, Indonesia, Oct 24-25, 2007. Retrieved February 15, 2010 from http://www.springerlink.com/index/q78020282234148r.pdf

Haykin, S. (2004). Feedforward neural networks: An introduction. Retrieved June 20, 2010 from: http://media.wiley.com/product_data/excerpt/19/04713491/0471349119.pdf

Joint Admissions Board. (2009). Guidelines to K.C.S.E. candidates on application for admission to public universities. Nairobi: University of Nairobi.

Joint Board should review mode of admitting university students. (2009, August 06). Daily Nation, p. 12. Retrieved March 24 from http://multimedia.marsgroupkenya.org/?StoryID=262623

Lin, Y., Zhang, Z., \& Thibault, J. (2009). Comparison of experimental design using neural networks. The Canadian Journal of Chemical Engineering, 87(6), 965-971.

Munavu, R., Ogutu, D., \& Wasanga, P. (2008). Sustainable articulation pathways and linkages between upper secondary and higher education in Africa. Association for the Development of Education in Africa

Rosini, P. (2000). Using expert systems and artificial intelligence for real estate forecasting. Sixth Annual Pacific-rim Real Estate Society Conference, Sydney, Australia, 24-27 January 2000. Retrieved February 18, 2010 from http://www.prres.net/proceedings/proceedings2000/P6A2.pdf

Rumelhart, D. E., Hinton, G. E., \& Williams, R. J. (1986). Learning internal representations by error propagation. In D. E. Rumelhart, \& J. L. McClelland (Eds.), Parallel distributed processing (pp. 318-362). MIT Press

Sodiya, S. A., \& Onashoga, S. A. (2009). Components-based access control architecture. Issues in Informing Science and Information Technology, 6, 669-705. Retrieved from http://iisit.org/Vol6/IISITv6p699-706Sodiya650.pdf

Werbos, P. J. (1974). Beyond regression: New tools for prediction and analysis in the behavioral sciences. (Doctoral dissertation, Harvard University, Cambridge, MA)

Zhang, G. P. (2000). Neural networks for classification: A survey. IEEE Transactions on Systems, Man, and Cybernetics - part c: Applications and Reviews, 30(4), 451. Retrieved February 12, 2010 from http://vis.lbl.gov/ romano/mlgroup/papers/neural-networks-survey.pdf 


\section{Biographies}

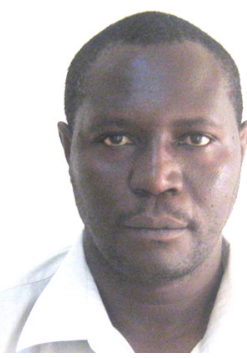

Mr. Franklin Wabwoba is a lecturer at Masinde Muliro University of Science and Technology in the Computer Science department. He holds a Master of Science (Computer Applications) degree from Kenyatta University; Endorsement (Educational Management) from University of South Africa and Bachelor of Education (science: Mathematics and Computer Science) degree from Egerton University and is pursuing a PhD (Information Technology) programme at Masinde Muliro University of Science and Technology. He has taught Computer Science and Information Technology courses for over twenty years. He has ICT industrial experience having worked with Mumias Sugar Company. He has presented several papers in scientific conferences and has a number of publications. He has a strong research interest in the impact of ICT applications on the community, integration of ICT into education, and green ICT.

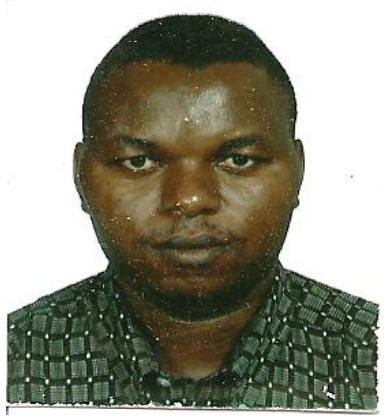

Mr. Fullgence M. Mwakondo is a graduate assistant at Mombasa polytechnic University College in the Department of Computer Science. He has over ten years of teaching experience. He holds a Bachelor of Science (Mathematics and Computer Science) from Jomo Kenyatta University of Agriculture and Technology. He is currently a Master of Science (Information Technology) student at Masinde Muliro University of Science and Technology in the Department of Computer Science. He has presented a number of papers at conferences. 